\title{
Unphysical properties of the static quark-antiquark four-point correlator in Landau gauge
}

\author{
Jeff Greensite and Evan Owen \\ Physics and Astronomy Department, San Francisco State University, \\ San Francisco, California 94132, USA
}

(Received 15 November 2018; published 14 January 2019)

\begin{abstract}
We consider the four point connected correlator representing a static quark-antiquark pair separated by a spatial distance $R$, propagating for a Euclidean time $T$. This function is computed by lattice Monte Carlo in $\mathrm{SU}(2)$ pure gauge theory at lattice couplings $\beta=2.2$ and $\beta=2.5$ in both Coulomb and Landau gauges. The Coulomb gauge correlator is well behaved, and is dominated at large $T$ by a state whose energy grows linearly as $\sigma R$, with $\sigma$ the known asymptotic string tension. The connected correlator in Landau gauge behaves differently. At intermediate $R$ there is clear evidence of a linear potential, but the corresponding string tension extrapolates to zero at large $T$. At large $R$ the connected correlator becomes negative; moreover there are strong finite size effects. These numerical results suggest that unphysical states dominate the large Euclidean time behavior of this Landau gauge correlator.
\end{abstract}

DOI: 10.1103/PhysRevD.99.014506

\section{INTRODUCTION}

It is well known that the Landau gauge gluon propagator, as computed in lattice Monte Carlo simulations, violates reflection positivity $[1,2]$ and this fact is viewed by some as indicative of gluon confinement. Then it is of interest to ask whether anything similar happens in Landau gauge quarkantiquark connected four point functions. One expects that there are poles in the connected four point functions corresponding to single meson states, and these poles should have a positive residue. This is, in fact, the starting point of the Bethe-Salpeter approach. But equal-times quarkantiquark operators, at distinct spatial points, do not create Becchi-Rouet-Stora-Tyutin (BRST) invariant states, and in any case both BRST invariance and reflection positivity are problematic in Landau gauge at the non-perturbative level, as we will discuss further below. So there is at least a possibility that Landau gauge quark antiquark four point functions exhibit unphysical behavior at large spacetime separations.

To investigate this possibility, we simplify matters as much as possible. We consider only the four point functions corresponding to static quarks and antiquarks with spatial separation $R$, evolving for a Euclidean time $T$, evaluated in pure $\mathrm{SU}(2)$ gauge theory. Since the quarks are static, this boils down to evaluating the connected correlator of Wilson lines

Published by the American Physical Society under the terms of the Creative Commons Attribution 4.0 International license. Further distribution of this work must maintain attribution to the author(s) and the published article's title, journal citation, and DOI. Funded by SCOAP ${ }^{3}$.

$$
\begin{aligned}
G(R, T) & \left.\left.=\frac{1}{2}\left\langle L_{T}^{a b}(\boldsymbol{x}) L_{T}^{\dagger b a}(\boldsymbol{y})\right]\right\rangle-\frac{1}{2}\left\langle L_{T}^{a b}(\boldsymbol{x})\right\rangle\left\langle L_{T}^{\dagger b a}(\boldsymbol{y})\right]\right\rangle \\
& =\left\langle\frac{1}{2} \operatorname{Tr}\left[L_{T}(\boldsymbol{x}) L_{T}^{\dagger}(\boldsymbol{y})\right]\right\rangle-\left\langle\frac{1}{2} \operatorname{Tr} L_{T}(\boldsymbol{x})\right\rangle^{2},
\end{aligned}
$$

where $L_{T}(\boldsymbol{x})$ is a timelike Wilson line on the lattice of length $T$, i.e.,

$$
L_{T}(\boldsymbol{x})=U_{4}(\boldsymbol{x}, 1) U_{4}(\boldsymbol{x}, 2) \ldots U_{4}(\boldsymbol{x}, T),
$$

and we have used the fact that, as a consequence of the remnant symmetry under spacetime independent gauge transformations $g(\boldsymbol{x}, t)=g$ which exists in Landau gauge

$$
\left\langle L_{T}^{a b}(\boldsymbol{x})\right\rangle=\frac{1}{2}\left\langle\operatorname{Tr} L_{T}\right\rangle \delta^{a b} .
$$

We note that in Coulomb gauge there is a remnant symmetry under time-dependent gauge transformations $g(\boldsymbol{x}, t)=g(t)$, and as a result

$$
\left\langle L_{T}^{a b}(\boldsymbol{x})\right\rangle=0 .
$$

In the large Euclidean time limit, $G(R, T)$ in Coulomb gauge should be dominated by the lowest energy eigenstate of the Coulomb gauge Hamiltonian $H_{\text {Coul }}$ containing a static quark-antiquark pair, while $G(R, T)$ in Landau gauge should likewise be dominated by the lowest energy eigenstate of the BRST Hamiltonian $H_{\mathrm{BRST}}$. But are these the same states? That is the question which we will try to address here numerically. 


\section{RESULTS}

Gauge-fixing is accomplished by the standard overrelaxation method, which applies, in each gauge-fixing sweep, an (over-relaxed) gauge transformation at each site, aiming to maximize the quantity

$$
R=\sum_{\boldsymbol{x}} \sum_{i=1}^{d} \operatorname{Tr}\left[U_{i}(x)\right],
$$

where $d=3$ and $d=4$ for Coulomb gauge and Landau gauge respectively. After each gauge-fixing sweep we calculate the fractional reduction in $R$ compared to the previous sweep. The gauge-fixing loop ends when the fractional reduction in $R$ falls below $10^{-10}$.

\section{A. Coulomb gauge}

We begin with results for $G(R, T)$ in Coulomb gauge. This type of calculation is not really new; the first results of this kind were obtained in Ref. [3], and later in [4,5]. We have included them here in order to make a comparison with the Landau gauge results to follow.

At large $R, T$ the $G(R, T)$ correlator is expected to be well approximated by a sum of terms falling exponentially with both $R$ and $T$. As an ansatz to extract an "effective" string tension $\sigma(T)$ at fixed $T$, we consider fitting the large $R$ data to a single exponential

$$
G(R, T) \approx c(T) e^{-\sigma(T) R T} .
$$

Assuming this gives a good fit to $G(R, T)$, we can then extrapolate $\sigma(T)$ to $T \rightarrow \infty$, where it is expected to converge to the usual asymptotic string tension.

However, on the lattice we must allow for periodic boundary conditions. If the quark-antiquark separation is parallel to the $x, y$ or $z$ axes, and the lattice is $N_{s}$ spacings in any of the space directions, then it is better to fit $G(R, T)$ to

$$
G(R, T) \sim c(T)\left(e^{-\sigma(T) R T}+e^{-\sigma(T)\left(N_{s}-R\right) T}\right) .
$$

By fitting the logarithm of the data for $G(R, T)$ vs $R$, at large $R$ and fixed $T$, to the logarithm of the right-hand side of (7), we can extract the string tension $\sigma(T)$.

We have carried out these fits at $\beta=2.5$ on a $24^{3} \times 40$ lattice. The time asymmetry is actually irrelevant in our Coulomb gauge data, as can be seen by comparing $G(R, T)$ at $T=8$ computed on a $24^{3} \times N_{t}$ lattice with $N_{t}=24$ and $N_{t}=40$. The comparison is shown in Fig. 1, and it is clear that the difference due to increasing $N_{t}$ from 24 to 40 is essentially negligible, as one would expect. We have nevertheless carried out simulations at $N_{t}=40$ in order to compare the Coulomb gauge data with the Landau gauge data on the same lattice volume.

The fit of our data to Eq. (7) is illustrated for $G(R, T)$, again at $T=8$, in Fig. 2, where the fitting region was the

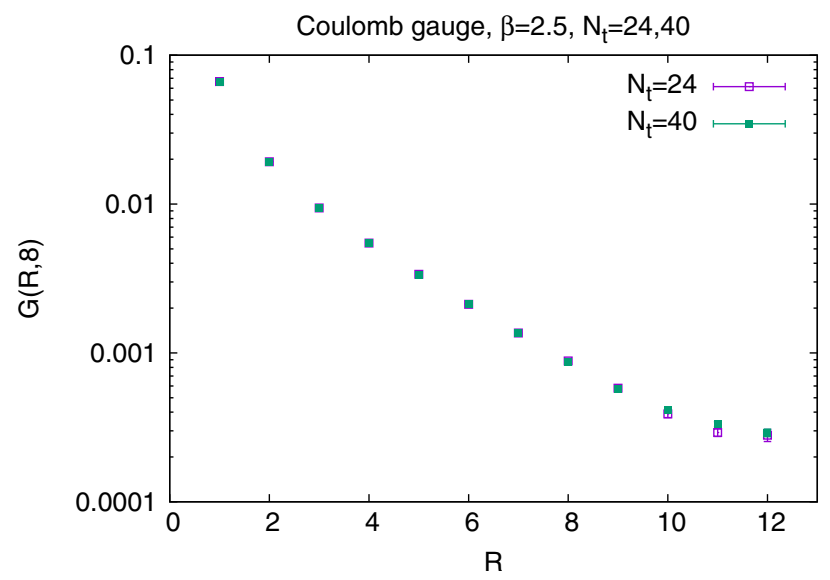

FIG. 1. Comparison of a logarithmic plot of $G(R, 8)$ vs $R$, for lattice volumes $24^{3} \times N_{t}$ and $N_{t}=24$ and $N_{t}=40$ lattice spacings. There is little difference in the two sets of data, as expected.

range $R>4$. The figure is representative of similar fits from $T=1$ to $T=9$. We plot the values of $\sigma(T)$ extracted from these fits in Fig. 3. The data is found to closely follow the curve

$$
\sigma(T)=\sigma_{\infty}+\frac{0.12}{T^{0.81}} \quad \text { where } \sigma_{\infty}=0.035(1)
$$

The asymptotic value $\sigma_{\infty}$ agrees within error bars with the $\mathrm{SU}(2)$ string tension at $\beta=2.5$ reported in [6].

So far there are no surprises. These results are consistent with expectations.

\section{B. Landau gauge}

In Fig. 4(a) we display $G(R, T)$ vs $R$ in Landau gauge at $T=3$ and an intermediate coupling strength of $\beta=2.2$. The lattice volume is $20^{4}$. A closeup of the data at separations $R \geq 3$ is shown in Fig. 4(b). From this figure

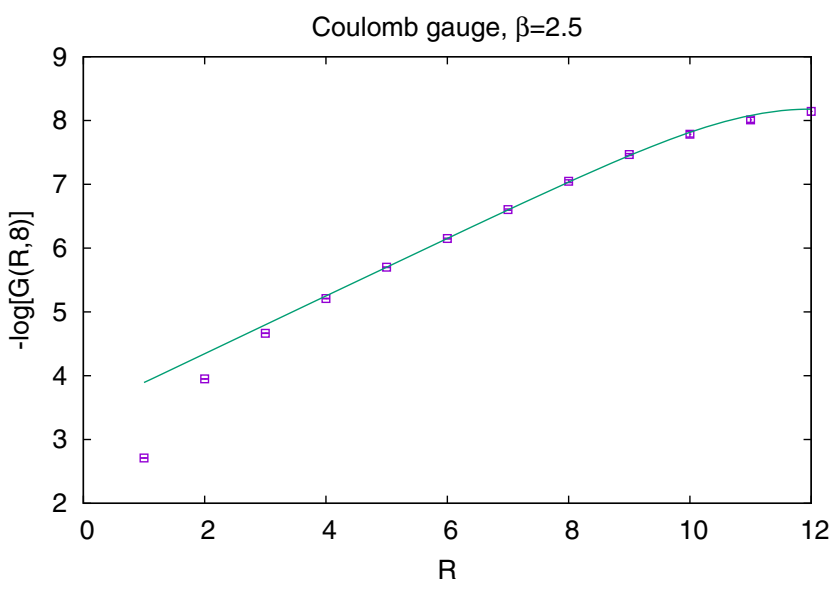

FIG. 2. Best fit of $-\log \left[\right.$ Eq. (7)], with $N_{s}=24$, to the data for $-\log [G(R, 8)]$. 


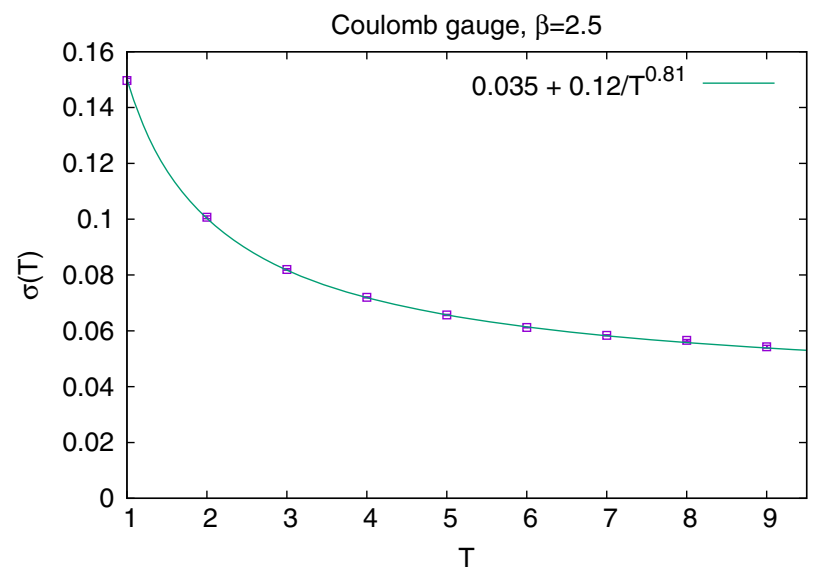

FIG. 3. String tension $\sigma(T)$ vs $T$, together with a best fit. The data extrapolates to the known asymptotic string tension of $\sigma=0.035$ in lattice units.

it is clear that the correlator violates positivity from $R=4$ onwards. We find a similar positivity violation in all plots of $G(R, T)$ vs $R$ at all $T$. For comparison we show in Fig. 5

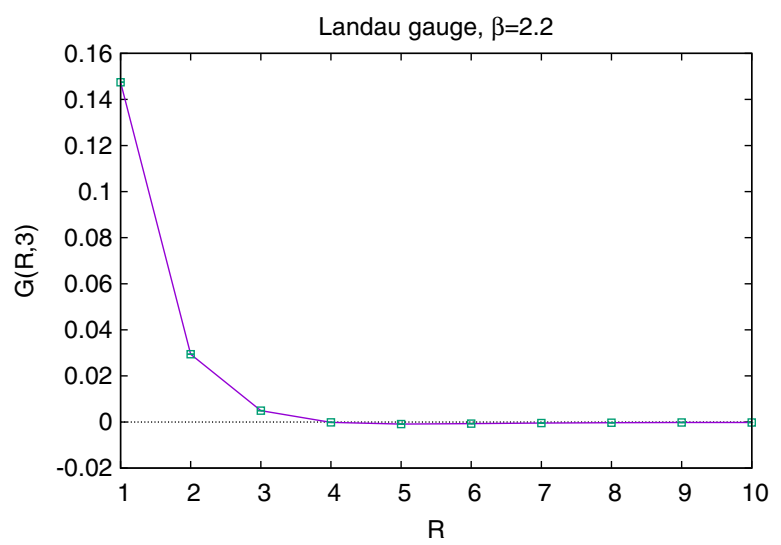

(a)

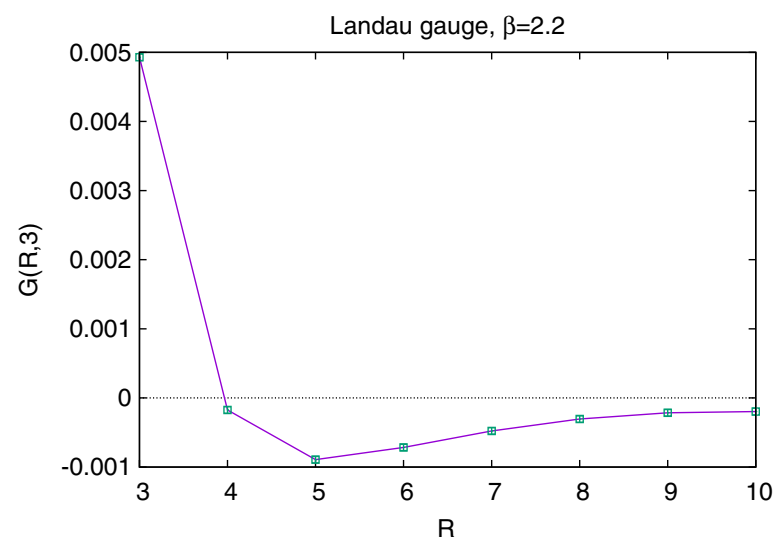

(b)

FIG. 4. Landau gauge connected correlator $G(R, T)$ vs $R$ at $\beta=2.2$ and $T=3$ on a $20^{4}$ lattice. (a) $G(T, 4)$ in the full range of $R$. (b) closeup in the range $R>2$. Note the violation of positivity in this range.

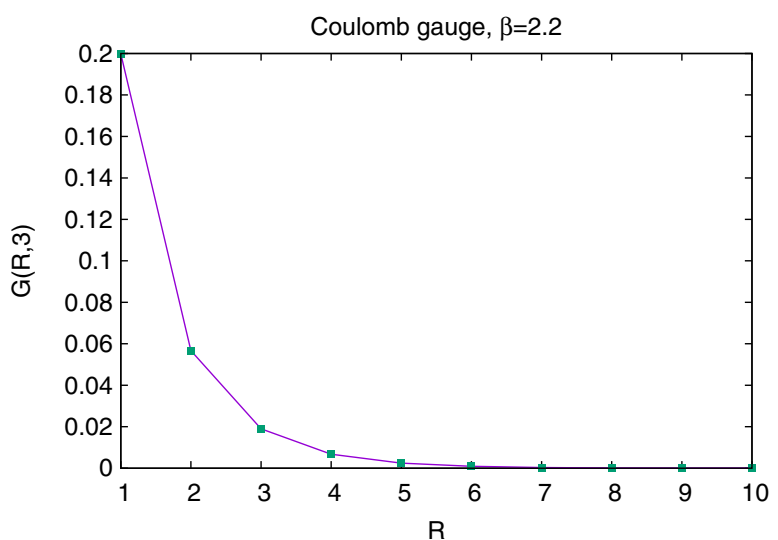

(a)

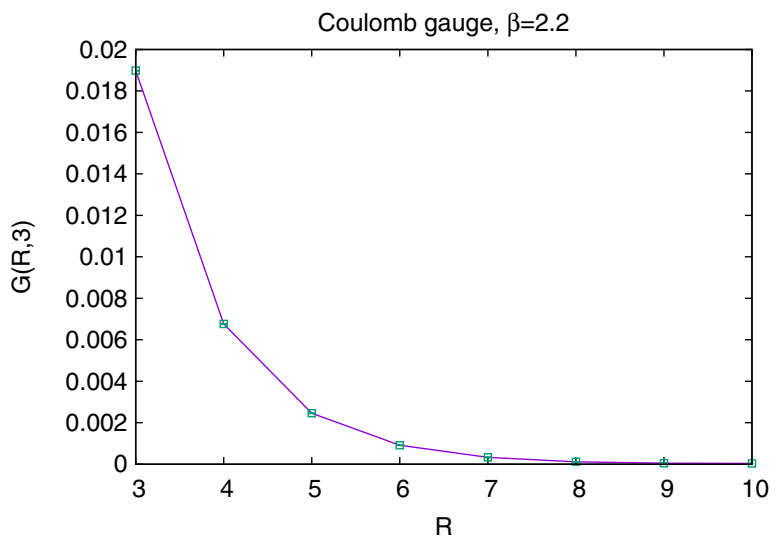

(b)

FIG. 5. Same as Fig. 4, but in Coulomb gauge.

the same plots at the same $\beta=2.2$ and lattice volume in Coulomb gauge.

At first sight this positivity violation seems to disappear at $\beta=2.5$. In Fig. 6 we display $G(R, T)$ at $T=6$, shown here on a logarithmic scale. It is clear that for $3 \leq R \leq 9$ the

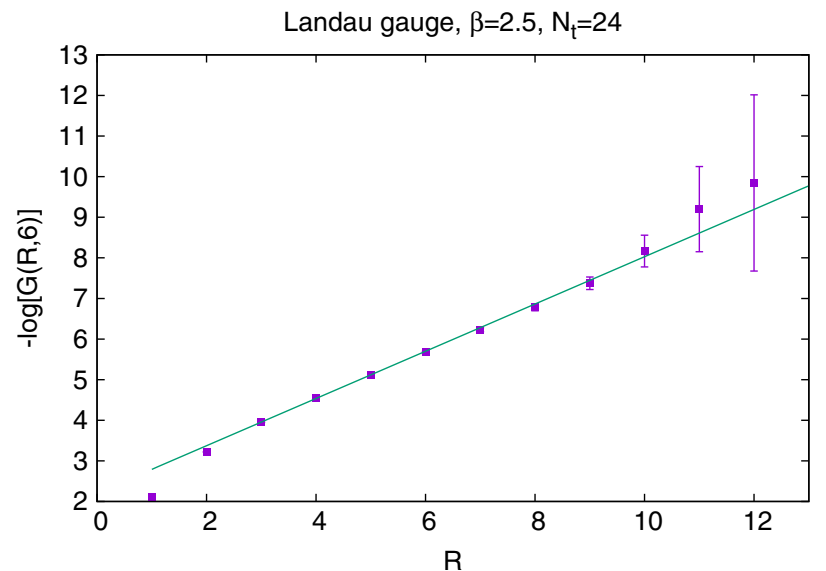

FIG. 6. Data for $-\log G(R, 6)$ vs $\mathrm{R}$ in Landau gauge at $\beta=2.4$ on a $24^{4}$ lattice, together with a linear fit through the larger $R$ values. 
data on a log plot is fit pretty well by a straight line, and this holds true for all $T$ up to $T=12$. Therefore, at $\beta=2.5$ on a $24^{4}$ lattice, we can follow the previous procedure in Coulomb gauge, and extract a $T$ dependent string tension $\sigma(T)$ from a fit of the data to

$$
G(R, T) \approx e^{-\sigma(T) R T} \quad R \geq 3
$$

Figure 7 is a plot of $\sigma(T)$ vs $T$ on a log-log plot. Unlike Coulomb gauge, the data is fit fairly well by

$$
\sigma(T) \approx \frac{0.587}{T}
$$

which means that $\sigma(T)$ extrapolates to zero as $T \rightarrow \infty$. The implication is that the Green's function is dominated, at large Euclidean times, by a state with zero string tension, i.e., an unphysical state.

We notice, however, that in Fig. 6 and in all other plots of $G(R, T)$ vs $R$ at constant $T$, there is no evidence of the "flattening out" of the data at the largest two or three values of $R$, which would have been expected due to periodic boundary conditions. In fact, and in contrast to Coulomb gauge, the data points at $R=10,11$ seem to even lie above the straight line fit, albeit there are large error bars. To investigate this further, we have increased the length of the lattice in the time direction to $N_{t}=30$ and $N_{t}=40$, while keeping the extension in the space directions fixed at 24 lattice spacings. When we do that, we find that the positivity violation found at $\beta=2.2$ reappears in $G(R, T)$ at all $T$ for $R>8$, as shown, e.g., in Fig. 8 at $T=8$. Evidently, apart from positivity violation, the Landau gauge correlator is subject to severe finite size effects.

In an intermediate range of $3 \leq R \leq 7$, we still observe on the $24^{3} \times 40$ lattice a linear rise in $-\log [G(R, T)]$ vs $R$, as seen in Fig. 9, and this allows us to extract the effective string tension $\sigma(T)$ of the corresponding potential in this

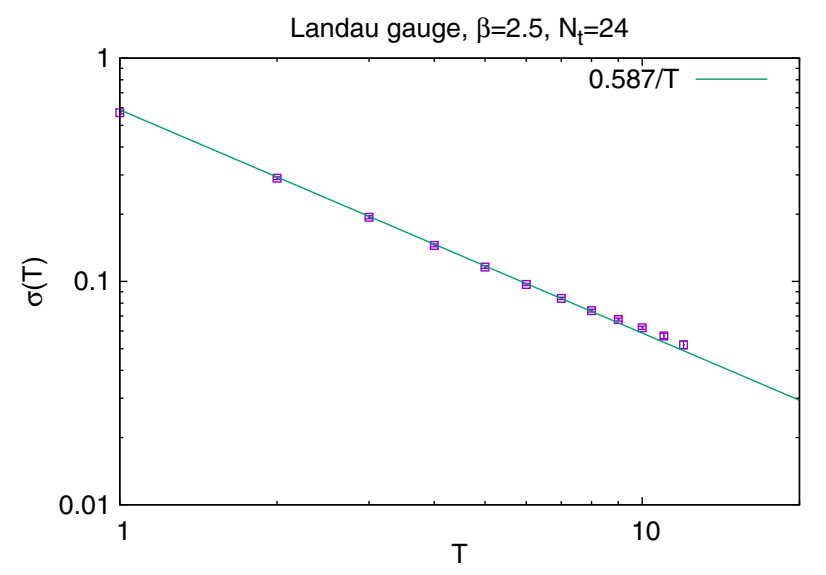

FIG. 7. String tension in Landau gauge, in lattice units, at $\beta=2.5$, obtained from fits to $G(R, T)$ data obtained on a $24^{4}$ lattice volume.

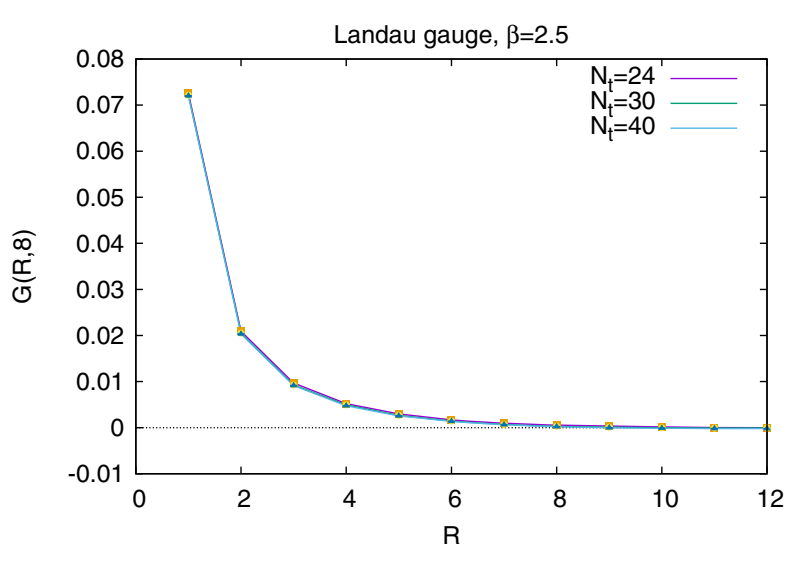

(a)

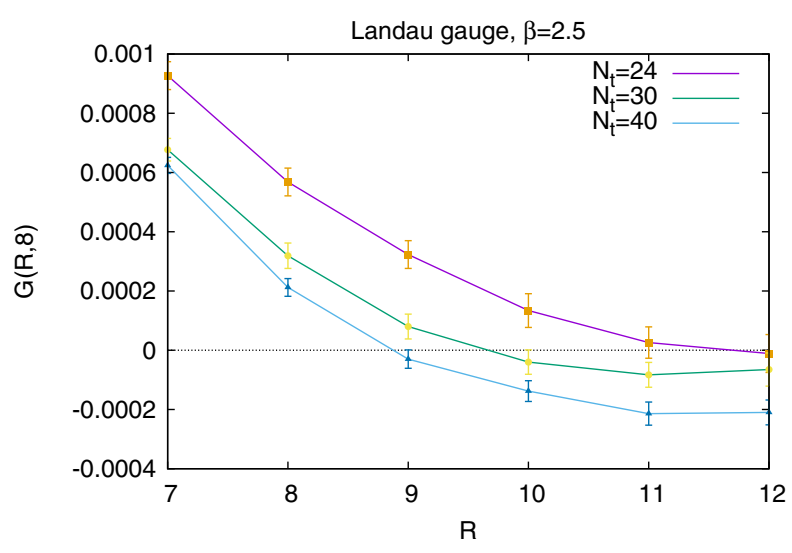

(b)

FIG. 8. Landau gauge connected correlator $G(R, T)$ vs $R$ at $\beta=2.5$ and $T=8$ on a $24^{3} \times N_{t}$ lattice, with $N_{t}=24,30,40$. (a) $G(T, 8)$ in the full range of $R$. (b) closeup in the range $R>6$, where we observe positivity violation at $N_{t}=30,40$.

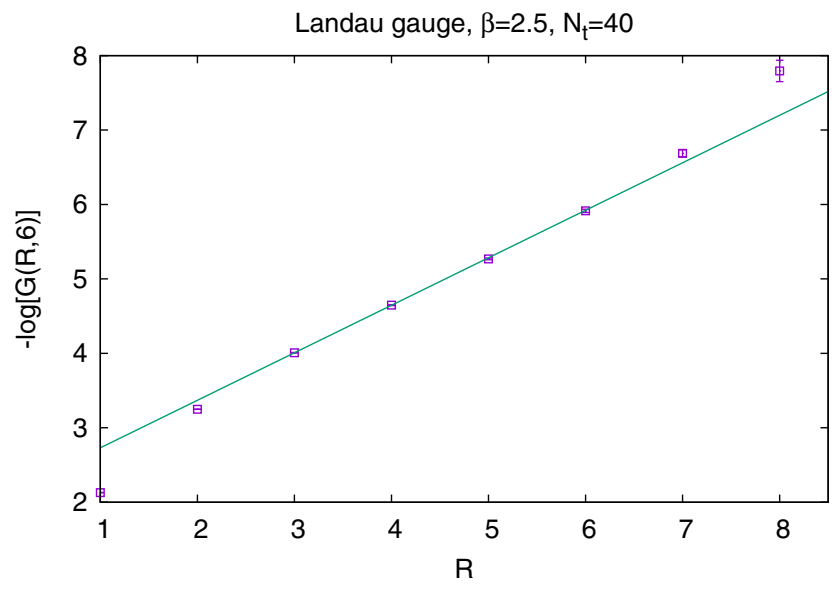

FIG. 9. Data for $-\log G(R, 6)$ vs $\mathrm{R}$ in Landau gauge at $\beta=2.4$ on a $24^{3} \times 40$ lattice, together with a linear fit in the range $3 \leq R \leq 7$. Note that $G(R, T)<0$ for $R>8$, so those points cannot be displayed in this figure. 


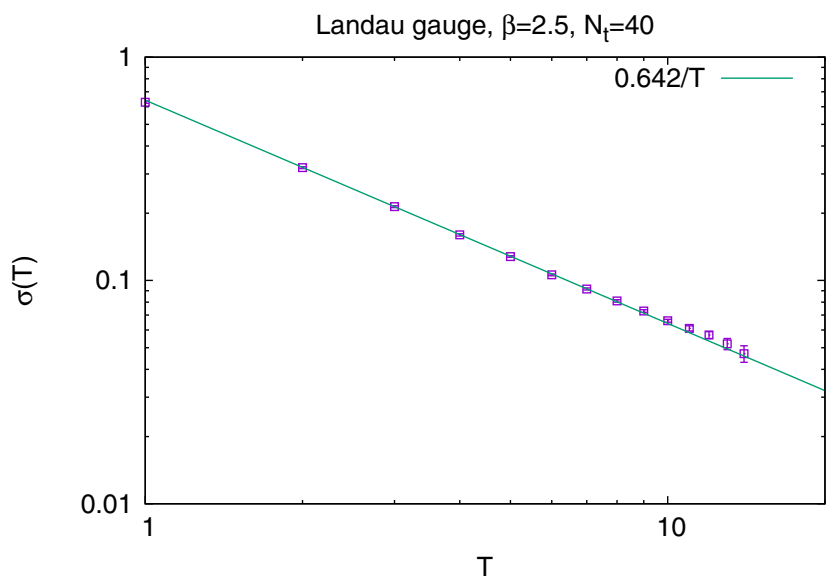

FIG. 10. String tension in Landau gauge, in lattice units, at $\beta=2.5$, obtained from fits to $G(R, T)$ data obtained on a $24^{3} \times 40$ lattice volume. In this case the string tension is extracted from fits to a restricted range $3 \leq R \leq 7$, due to the nonpositivity of the correlator at large $R$.

range. That string tension is plotted vs $T$ on a log-log scale in Fig. 10, and is fairly well fit by

$$
\sigma(T)=0.642 / T \text {. }
$$

As on the $24^{4}$ lattice volume, this string tension formally extrapolates to zero at $T \rightarrow \infty$, which of course is the wrong answer for the energy of a physical state containing a static quark-antiquark pair. However, we must note that for $T>12$ the nonpositivity affects data points down to $R=6$, and we do not feel justified in extracting a string tension from only three data points.

\section{Related work}

Ours is not the first study of the product of Wilson lines $\left\langle\operatorname{Tr}\left[L_{T}(\boldsymbol{x}) L_{T}^{\dagger}(\boldsymbol{y})\right]\right\rangle$ in Landau gauge. Iritani and Suganuma [7] have computed this quantity in $\mathrm{SU}(3)$ lattice gauge theory, and they have noted that because this quantity tends at large $R=|\boldsymbol{x}-\boldsymbol{y}|$ to a constant (i.e., the disconnected part), confining behavior cannot appear in this observable. The connected correlator was not computed in this reference, and therefore positivity violation, which is only seen in the connected Green's function, was not observed.

In a different study, Bicudo et al. [8] computed the quantity $\left\langle\operatorname{Tr}\left[P(\boldsymbol{x}) P^{\dagger}(\boldsymbol{y})\right]\right\rangle$ in Landau gauge, where $P(\boldsymbol{x})$ is a Polyakov line. Of course the gauge invariant correlator is $\left\langle\operatorname{Tr}[P(\boldsymbol{x})] \operatorname{Tr}\left[P^{\dagger}(\boldsymbol{y})\right]\right\rangle$, and for the motivation for the gaugedependent observable we must refer to the cited references. Bicudo et al. do not see any positivity violation in this operator, and in this case the reason is that the disconnected part, which is gauge invariant, vanishes in the confined phase. Since positivity violation comes about only after subtracting the disconnected part, the absence of positivity violation in this case is not surprising, and because the disconnected part vanishes, a string tension is a possibility.
Bicudo et al. do in fact find a string tension, as do we in the connected correlator $G(R, T)$ at fixed $T$. These authors work mainly in the confined phase, and mainly at fixed $T=N_{t}=12$, and find a string tension at various lattice couplings which is $30 \%$ smaller than the corresponding asymptotic string tension. This result is compatible with our findings; one expects zero string tension for $G(R, T)$ only in the $T \rightarrow \infty$ limit. Moreover, any string tension less than the asymptotic string tension (which is the minimum possible for a static quark-antiquark pair), is a clear indication, in our view, of the presence of unphysical states. It is difficult, however, to make any quantitative comparison with our work, partly because of the different gauge groups [SU(3) vs $\mathrm{SU}(2)]$, but also because our Wilson lines do not wind all the way through the periodic lattice, but stop at $T=N_{t} / 2$.

\section{LANDAU GAUGE AND ITS DISCONTENTS}

Obviously it would be wrong to conclude, from the nonpositivity of $G(R, T)$ in Landau gauge, that quarkantiquark bound states are absent in the spectrum. The problem is more likely due to the fact that the relevant quark-antiquark operator in Landau gauge is not a BRST singlet, and moreover that BRST symmetry is itself problematic at the nonperturbative level.

If the state created by the massive quark-antiquark creation operator $\bar{\psi}^{+a}(\boldsymbol{x}, t) \psi^{+a}(\boldsymbol{y}, t)$ is not annihilated by the BRST charge operator in a covariant gauge, then it is not a physical state. It may have an overlap with physical states, but there may also be non-negligible overlaps with negative norm and other unphysical states. In addition, BRST symmetry on the lattice is subject to the $0 / 0$ problem pointed out by long ago by Neuberger [9]. Let

$$
Z=\int D U D \bar{c} D c e^{-\left(S+S_{g f}\right)},
$$

where $S_{g f}$ is the standard BRST gauge-fixing term in a covariant gauge. Then, as shown in [9], it follows that $Z=0$. This is also true if a BRST invariant operator is inserted in the integrand, hence the expectation value of any such observable is formally $0 / 0$. The problem has to do with a summation over Gribov copies in covariant gauges, which contribute to the functional integral with both positive and negative signs. On the lattice, the gauge-fixing procedure restricts the evaluation to Gribov copies within the first Gribov horizon; i.e., to gauge copies which contribute to the expectation values with only positive sign. But this restriction itself breaks BRST invariance, as shown numerically by Cuccieri et al. [10].

In the absence of BRST invariance, even the usual assumptions underlying reflection positivity are suspect. Take, for example, the case of the Landau gauge gluon propagator. What is actually computed on the lattice is the expectation value 


$$
D_{\mu \nu}^{a b}(x-y)=\left\langle\left[G_{L} \circ A\right]_{\mu}^{a}(x)\left[G_{L} \circ A\right]_{\nu}^{b}(y)\right\rangle,
$$

where $G_{L}$ is a gauge transformation which takes the gauge field into some copy of Landau gauge within the first Gribov horizon. But $G_{L}$ is obviously nonlocal in time, which violates one of the assumptions underlying the usual proof of reflection positivity, and this is perhaps the reason for the observed lack of positivity in the Landau gauge gluon propagator. If the gauge copies are not restricted to the Gribov region, then one might argue that time nonlocality could be eliminated at the price of introducing ghost fields, i.e.,

$D_{\mu \nu}^{a b}(x-y)=\frac{1}{Z} \int D A_{\mu} D c D \bar{c} A_{\mu}^{a}(x) A_{\nu}^{b}(y) \exp \left[-\left(S+S_{g f}\right)\right]$.

But this strategy, as already mentioned, runs right into the Neuberger $0 / 0$ problem. For Landau gauge, and for covariant gauges in general, the choice is to either break BRST explicitly, or face the $0 / 0$ problem.

Neither option is attractive. In lattice simulations the choice is to break BRST symmetry explicitly, which at least produces a well-defined answer. But perhaps it is then not surprising that the resulting four-point Euclidean Green's functions for massive quark antiquark states are found to exhibit unphysical behavior.

\section{CONCLUSIONS}

We have found that the Coulomb gauge four point function $G(R, T)$ corresponding to creation and destruction of a static quark antiquark pair, separated by a spatial distance $R$, behaves as expected: the correlator falls off exponentially with $R T$ as in Eq. (7), with an effective string tension $\sigma(T)$ extrapolating, as $T \rightarrow \infty$, to the known asymptotic string tension. In contrast, the corresponding connected two point function in Landau gauge exhibits two pathologies. First, while $G(R, T)$ does fall off exponentially with $R$ for an intermediate range of $R$, the string tension $\sigma(T)$ appears to extrapolate to zero at large $T$, indicative of dominance by an unphysical state. Second, at large $R$, the connected four point function is negative, likewise indicating dominance by negative norm states.

The first question is whether these types of unphysical behavior persist in quark-antiquark four point functions for quarks with finite mass, and this will be the next issue to investigate. Assuming that unphysical behavior persists at finite mass, which we believe is likely, the next question is: do our results pose a problem for the existing DysonSchwinger (DS) and functional renormalization group (FRG) approaches to Landau gauge-fixed QCD, both of which entail the nonperturbative computation of irreducible n-point functions?

It is difficult to provide a definite answer at the moment. Some studies, e.g., [11], which combine the DysonSchwinger and Bethe-Salpeter equations, have had quite some success in treating the low-lying hadron spectrum. It may be that these approaches somehow avoid the issue of unphysical states, perhaps by concentrating on n-point functions in the neighborhood of physical poles. Is it then possible, within the DS and FRG schemes, to also uncover the presence of unphysical states in the four point correlation functions? Or are such states necessarily absent in these approaches? Perhaps the truncations which are inevitable in the DS and FRG schemes lose information about unphysical states? (If so, what else might be lost?) We do not know the answers to any of these questions, but we believe they may be worth further investigation.

\section{ACKNOWLEDGMENTS}

J.G. would like to acknowledge discussions with Christian Fischer and Jan Pawlowski. This work is supported by the U.S. Department of Energy under Grant No. DE-SC0013682.
[1] P. O. Bowman, U. M. Heller, D. B. Leinweber, M. B. Parappilly, A. Sternbeck, L. von Smekal, A. G. Williams, and J. Zhang, Phys. Rev. D 76, 094505 (2007).

[2] A. Cucchieri, T. Mendes, and A. R. Taurines, Phys. Rev. D 71, 051902 (2005).

[3] E. Marinari, M. L. Paciello, G. Parisi, and B. Taglienti, Phys. Lett. B 298, 400 (1993).

[4] J. Greensite and S. Olejnik, Phys. Rev. D 67, 094503 (2003).

[5] T. Heinzl, K. Langfeld, M. Lavelle, and D. McMullan, Phys. Rev. D 76, 114510 (2007).

[6] G. S. Bali, K. Schilling, and C. Schlichter, Phys. Rev. D 51, 5165 (1995).
[7] T. Iritani and H. Suganuma, Phys. Rev. D 83, 054502 (2011).

[8] P. Bicudo, N. Cardoso, O. Oliveira, and P. J. Silva, Proc. Sci. LATTICE2011 (2011) 300; N. Cardoso, P. Bicudo, P. J. Silva, and O. Oliveira, Acta Phys. Pol. B Proc. Suppl. 5, 1135 (2012).

[9] H. Neuberger, Phys. Lett. B 183, 337 (1987).

[10] A. Cucchieri, D. Dudal, T. Mendes, and N. Vandersickel, Phys. Rev. D 90, 051501 (2014).

[11] G. Eichmann, H. Sanchis-Alepuz, R. Williams, R. Alkofer, and C. S. Fischer, Prog. Part. Nucl. Phys. 91, 1 (2016). 